Applied Remote Sensing

RemoteSensing.SPIEDigitalLibrary.org

\title{
2017 List of Reviewers
}


The Journal of Applied Remote Sensing would like to sincerely thank the following individuals who served as reviewers in 2017. The success of our publication hinges on the voluntary contributions of time and energy put forth by these professionals.

\begin{tabular}{|c|c|}
\hline James Aanstoos & Behnaz Bigdeli \\
\hline Isaac Abazu & Gokhan Bilgin \\
\hline Elfatih Abdel-Rahman & Hamidullah Binol \\
\hline Nicola Acito & Marcelo Biudes \\
\hline Zakaria Adiri & Andrew Bodkin \\
\hline Gabriela Afrasinei & Duncan Boiyo \\
\hline K.M. Agrawal & Stefania Bonafoni \\
\hline Noordin Ahmad & Frederic Borne \\
\hline Saeed Ahmad & Emmanuel Boss \\
\hline Jiaqiu Ai & Robert Bracken \\
\hline Oluibukun Ajayi & Alexander Braun \\
\hline Gholamreza Akbarizadeh & Maximilian Brell \\
\hline Hayder AI-Salihi & Fabio Breunig \\
\hline Miguel Albuquerque & Andrey Bril \\
\hline Thomas Alexandridis & Joshua Broadwater \\
\hline Abebe Ali & Olga Brovkina \\
\hline Mohammad AIShabi & Krishna Buddhiraju \\
\hline Cihan Altuntas & Merv Budge \\
\hline Shrinidhi Ambinakudige & Alessandra Budillon \\
\hline Clark Amerault & Mariko Burgin \\
\hline Ruhul Amin & Carlos Cabo \\
\hline Shahid Amjad & Arturo Cadena \\
\hline Daoxiang An & Mingyong Cai \\
\hline Marios N. Anagnostou & Marcello Caleffi \\
\hline Derek T. Anderson & Petya Campbell \\
\hline Eric Anderson & Isidro Campos \\
\hline Paul Aplin & Changyong Cao \\
\hline Francisco Arguello & Jinde Cao \\
\hline Abd. Rahman As-syakur & Facundo Carmona \\
\hline Afshin Asefpour Vakilian & Oliver Cartus \\
\hline Jan Audenaert & Nicolas Cezard \\
\hline Stefan Auer & Sumit Chakravarty \\
\hline Dogan Aydal & Chee Seng Chan \\
\hline Rodolfo B. Arango & Kwing Chan \\
\hline Alessio Bacci & Ni-Bin Chang \\
\hline Charles Bachmann & Sushank Chaudhary \\
\hline Kaixu Bai & Chen Chen \\
\hline Xiaolin Bai & Chi Chen \\
\hline Shahbaz Baig & Jin Chen \\
\hline John Ball & Lei Chen \\
\hline Eric Balster & Lin Chen \\
\hline Biplab Banerjee & Ming Chen \\
\hline Georg Bareth & Yushi Chen \\
\hline William Barnes & Zhikang (Ken) Chen \\
\hline Terry Bartlett & Dongcai Cheng \\
\hline Christian Bauer & Gong Cheng \\
\hline Vivek Bawa & Tao Cheng \\
\hline Ebrahim Bedeer & Marco Chini \\
\hline Alireza Behrad & Jerzy Chmiel \\
\hline Amin Beiranvand Pour & Yunjae Choung \\
\hline Yury Bekhtin & Cornell S.L. Chun \\
\hline Mariana Belgiu & James Churnside \\
\hline Chris Bellman & Ryan Close \\
\hline Bhojaraja Benkipura Eshwarappa & Emanuel Coelho \\
\hline Segio Bernabe & Adriaan Combrink \\
\hline Partha Bhattacharjee & Simona Consoli \\
\hline Avik Bhattacharya & Giovanni Crosta \\
\hline Xiaoyong Bian & Ovidiu Csillik \\
\hline
\end{tabular}

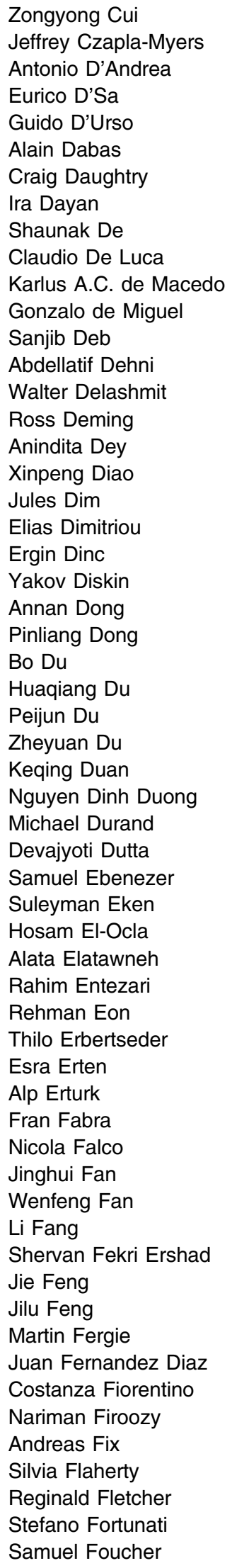


Michael Foumelis

Kelly Franklin

Mariusz Frukacz

Douglas Fuller

Tamon Fumoto

Frederic Galland

Lenio Galvao

Fabio Gama

Paolo Gamba

Feng Gao

Lianru Gao

Angel Garcia

Andrea Garzelli

Carol Garzon-Lopez

Andrew Gatesman

Sun Genyun

Izidor Gertner

Morteza Ghahremani

Reham Gharbia

Sachim Ghude

Alex Gilerson

Fulvio Gini

loannis Gitas

Anatoly Gitelson

Sertan Gokce

Maoguo Gong

Sundararaman G. Gopalakrishnan

Ben Gorte

Kirk Gossage

Tristan Goulden

Raffaele Grasso

Danielle Grogan

James H. Gruben

Lingjia Gu

Xiaodong $\mathrm{Gu}$

Arindam Guha

Subhanil Guha

Oguz Gungor

Ping Guo

Xiaojiang Guo

Xulin Guo

Dileep Gupta

Sharad Gupta

David Gwenzi

Christian Haas

Mohammed Hachama

Madeline Hack

Ronny Haensch

Alireza Hamedianfar

Wei Han

Xianwei Han

Yahong Han

Youkyung Han

Leonard Hanssen

Archit Harsh

Sayeh Hasan

Noordyana Hassan

Lakshminarayan Hazra

Lei $\mathrm{He}$

Tao He
Yuhong $\mathrm{He}$

D.C. Heinz

Johannes Heinzel

Mary Henry

Scott Hensley

Stephen Herbst

Stien Heremans

Derrick Herndon

Ittai Herrmann

Ryan Hersey

Eric Hetland

Haikel Hichri

Donald Hillger

Juergen Holfort

Saeid Homayouni

Annelies Hommersom

Seyed Hossein Hosseini Nourzad

Stephan Howden

Sheng-Jen Hsieh

Pai-Hui Hsu

Mou $\mathrm{Fa} \mathrm{Hu}$

Yonghong $\mathrm{Hu}$

Hong Huang

Wenjiang Huang

Yanbo Huang

Yong-Ren Huang

David Hulslander

E. Hunt

Chunlei Huo

Du Huynh

Paul Hwang

Patrick Hytla

Antonio lodice

Kengo lokibe

Javed lqbal

Muhammad Aminul Islam

Akira Iwasaki

Nasehe Jamshidpour

SiowWei Jaw

Satish Jayapal

Philip-Neri Jayson-Quashigah

Kun Jia

Xiuping Jia

Ruinian Jiang

Shuanggen Jin

Xiaoying Jin

Xin Jin

Linhai Jing

Eric H. Johnson

Akhil Kallepalli

Vipin Kamble

Nur Huseyin Kaplan

Shahid Karim

Mesut Kartal

Teerasit Kasetkasem

Dhanesh Kattipparambil Rajan

Jason Kaufman

Taskin Kavzoglu

Michal Kedzierski

Ronald Kemker
Ramesh Kestur

Saeed Khabbazan

Unmesh Khati

Kourosh Khoshelham

David Kim

Youngok Kim

Andy Kitchen

Joni Kluss

Kyle Knipper

Anders Knudby

Takumi Kobayashi

Dilek Koc-San

Ven Jyn Kok

Caitlin Kontgis

Xiaokang Kou

Matthew Kowalewski

Pedzisai Kowe

Andrey Krutikov

Krzysztof Kulpa

Amit Kumar

Anil Kumar

Karanam Kumar

Shashi Kumar

Vineet Kumar

Jitka Kumhalova

N. Kunihiro

Mehmet Kurum

Shunichi Kusano

Tiit Kutser

Geir Kvaran

F.L.

Pierre Lahaie

Salim Lamine

Charis Lanaras

Alexandre langlois

Kristofer Lasko

Daniel Lavigne

Minda Le

Vannhu Le

Dong-Cheon Lee

Ken Yoong Lee

Robert Lee

Ruibo Lei

Bob Lempkowski

Nathaniel Levitan

Bo Li

Dong Li

Erzhu Li

Honggui $\mathrm{Li}$

Hui Li

Jianwei Li

Jianxun Li

Jun $\mathrm{Li}$

Jun Li

Jun Li

Ming Li

Ning Li

Peijun Li

Shanshan Li

Wei Li 
Xiaofeng Li

Xinghua Li

Xingong Li

Xinwu Li

Xueyuan Li

Yang Li

Yanyan $\mathrm{Li}$

Yingkui Li

Yunying Li

Zhan Li

Zhaoqin $\mathrm{Li}$

Zhe Li

Xinlian Liang

Soo Chin Liew

Tang-Huang Lin

Eva Lindberg

Truong-Hong Linh

Bing Liu

Chenguang Liu

Hongying liu

Jianhong Liu

Jun Liu

Liangyun Liu

Lingling Liu

Shishi Liu

Sicong Liu

Xiaoye Liu

Yi Liu

Yonggang Liu

Yuling Liu

Zhandong Liu

Zhengjun Liu

Jurgen Lobert

Bharat Lohani

Jenny Lovell

Benjamin Lowry

Bing Lu

Xiaomei Lu

Chang Luo

Guido Luzi

Heng Lyu

Aidy M. Muslim

Chao Ma

Ronghua Ma

Xiaofeng Ma

Ghassan Maalouli

Mehdi Maanan

Mehdi Maboudi

Sean MacFaden

Troy Magney

Abhijit Mahalanobis

Amir Pasha Mahmoudzadeh

Stefan Maier

Konstantinos Makantasis

Eva Malinverni

Satyanarayana Malladi

Jordi Mallorqui

Antonino Maltese

Dimitris Manolakis

Chongyuan Mao
Javier Marcello-Ruiz

Adrian Mariano

Armando Marino

Andrea Marinoni

Tapas Martha

Gabriel Martin

Estibaliz Martinez

Anthony Martino

Antoine Masse

Aneesh Mathew

Stefania Matteoli

Newfel Mazari

Stephen McNeill

Mustapha Meftah

Alessandro Mei

Shaohui Mei

Tatiana Mendes Sussel

Fang Meng

Jihua Meng

Vineetha Menon

Nina Merkle

Olivier Merlin

Graciela Metternicht

Suresh Middinti

Elizabeth Middleton

Mario Minacapilli

Katarzyna Mirek

Kumar Vijay Mishra

Harvey Mitchell

Fan Mo

Helmi Mohd Shafri

Marcos Montes

Thomas Moranduzzo

Alexandra Morel

Wesley Moses

Dimitrios Moshou

P. Mukhopadhyay

Karthik Muthuswamy

Sudhagar Nagarajan

Laven Naidoo

Venkata Narra

Venkatanathan Natarajan

Said Nawar

Majid Nazeer

Rogerio Negri

lain Neil

Brian $\mathrm{Ng}$

C. Nichols

Doina Nicolae

Chunyue Niu

Yan Niu

Zheng Niu

Kazutoshi Noda

Imama Noor

Stefani Novoa

Ebenezer Nyadjro

Kenta Obata

Michael Obland

Ozben Onhon

Irena Orovic
Maria Fernanda Ortuno

Mohammed Ouali

Bing Ouyang

Asli Ozdarici-Ok

Mehmet Ozdogan

Jayesh Pabari

Todd Paciencia

Mary Pagnutti

Nima Pahlevan

Celia Paiva

Mahesh Pal

M. Palano

Dmitriy Paliy

Luca Pallotta

Yun Pan

Panos Panagos

Prem Pandey

Amit Kumar Pandit

Matteo Pardini

Panagiotis Partsinevelos

Debora Pastina

Swarnajyoti Patra

Audrey Paulus

Sergey Pavlov

William Pegau

Jifang Pei

Jian Peng

Jiangtao Peng

Liu Peng

Tao Peng

Zhenming Peng

Antonio Pepe

Said Pertuz

Dana Petcu

Hai Pham

Anh Thu Phan

Mark Phillips

Stuart Phinn

Shaohua Pi

Anthony Pinar

Joao Pompeu Pavanelli

Loredana Pompilio

Victor Pomponiu

Mangalraj Poobalasubramanian

David Porter

Richa Prajapati

Surya Prakash Pattanayak

Rajendra Prasad

G. Prates

Cameron Proctor

Xiaojun Qi

Gang Qiao

Lindi Quackenbush

Pablo Quesada Barriuso

Sudha Radhika

Karnam Raghunath

Mahmudur Rahman

Roozbeh Rajabi

Upendra Rajput

Nareenart Raksuntorn 
Desikan Ramakrishnan

Vinod Ramnath

Abel Ramoelo

Behnood Rasti

Daniel Raucoules

Mehul Raval

Francois Ravetta

Ann Raynal

Abdur Raziq

Karin Rebel

K. Reddy

Guoquan Ren

Ronald Resmini

Martina Ricko

Haris Riris

Nadia Rochdi

Victor Rodriguez-Moreno

Komeil Rokni

Ribana Roscher

Jens Rosebrock

Luke Rosenberg

Hang Ruan

Ana Ruiz-Constan

Nastaran Saberi

Dario Sacco

Amit Sahu

Masanori Saito

Federica Salvetti

Alim Samat

Fabrizio Santi

Asiya Sarinova

V. Sathiyamoorthy

Travis Sawyer

Michael Sayers

Stephen Schiller

Mitch Schull

Grant Scott

Imane Sebari

Umut Sefercik

Alfonso Senatore

Gowri Senthil

Donghoan Seo

Guy Serbin

Andres Serna

Mahdi Shabankareh

Reza Shah-Hoseini

Muhammad Shahzad

Muhammad Shakir

Hui Shao

Lakesh Sharma

Som Kumar Sharma

Vaibhav Sharma

Joseph Shaw

Vittala Shettigara

Shuo Shi

Zhenwei Shi

Moojoon Shin

Maxim Shoshany

Greg Showman

Yanmin Shuai
Till Sieberth

Fabricio Silva

Thiago Silva

Daniela Silva-Fuzzo

Elisabeth Simonetto

Pratap Singh

Ramesh Sivanpillai

Drazen Skokovic

Karl Skretting

Terrence Slonecker

Richard Smith

William Smith

Hilary E. (Ned) Snell

Foo Chong Soon

Jose Sotoca

Hari Shanker Srivastava

Daniele Stagliano

Michael Starek

Demetris Stathakis

Hongbo Su

Hongjun Su

Haibin Sun

Kang Sun

Qiang Sun

Robert Sundberg

Jurate Suziedelyte Visockiene

N. Kumar Swamy

Olga Sykioti

Muhammad Tahir

Fuyi Tan

Jing Tan

He Tang

Qingping Tao

Alireza Taravat

Gulsen Taskin

Martin Tauc

Ahmet Tekeli

Marivi Tello

Prasad Thenkabail

Mary Thornbush

R. Thriveni

Feng Tian

Ruiqi Tian

Sirui Tian

Zhiqiang Tian

Sonia Tomei

Alexander Tong

Hengjian Tong

Nathan Torbick

Alfonso Torres-Rua

Melanie Trudel

Chun-Ming Tsai

Song Tu

Esra Tunc Gormus

Eija Tuominen

Ashish Upadhyay

Kishor Upla

Ruben Usamentiaga

Mustafa Ustuner

Faruk Uysal
Juan Valdiviezo-Navarro

Freek van der Meer

Piet van Genderen

Adriaan Van Niekerk

Dmitry Vengertsev

Marie-Leen Verdonck

Jagath Vithanage

James Voogt

Benoit Vozel

Francesco Vuolo

Alaaddin Vural

Philippe Waldteufel

Jeff Walker

Chenxi Wang

Chi-Kuei Wang

Dong Wang

Haipeng Wang

Hongquan Wang

Jianyu Wang

Jun Wang

Ling Wang

Ran Wang

Robert Wang

Tianxing Wang

Xiaoyang Wang

Yong Wang

Yong Wang

Yonghui Wang

Zongming Wang

Lianhuan Wei

Pan Wei

Qi Wei

Zhihui Wei

Flora Weissgerber

Duminda Welikanna

XianBin Wen

Joseph White

Pramaditya Wicaksono

Rohan Wickramasuriya

Damian Wierzbicki

Aisheng Wu

Hongbo Wu

Junjie Wu

Yonghua Wu

Yuanfeng Wu

Junshi Xia

Donghui Xie

Huan Xie

Zhixiao Xie

Jingwei $X u$

Qizhi Xu

Manohar Yadav

Fuli Yan

Weidong Yan

Jian Yang

Ping Yang

Qian Yang

Taoli Yang

Wei Yang

Xiaohui Yang 
Yi Yang

Yingbao Yang

Dogukan Yavasli

Yunming Ye

Bhavani Kumar Yellapragada

Seokwon Yeom

Faliu Yi

Enes Yigit

B. Yigit Yildiz

Volkan Yilmaz

Fangfang $\mathrm{Yu}$

Xiaohui Yuan

Yuhendra Yusuf

Thomas Zajakowski

Yuebo Zha

Chengye Zhang

Chunhua Zhang

Guoqing Zhang
Hankui Zhang

Jiahua Zhang

Lei Zhang

Lifu Zhang

Miao Zhang

Qingyuan Zhang

Xiaoyang Zhang

Yi Zhang

Yifan Zhang

Yu Zhang

Yunhua Zhang

Zhiming Zhang

Jianhu Zhao

Shangmin Zhao

Wei Zhao

Yanyu Zhao

Yindi Zhao

Guang Zheng
Haiyong Zheng

Quanan Zheng

Shuiling Zheng

Xingming Zheng

Zezhong Zheng

Chengwei Zhou

Ji Zhou

Jun Zhou

Wenzuo Zhou

Xiran Zhou

Zeng-Guang Zhou

Feiya Zhu

Jie Zhu

Giuseppe Zibordi

Andrea Zingoni

Roghayeh Zoleikani

Bin Zou 